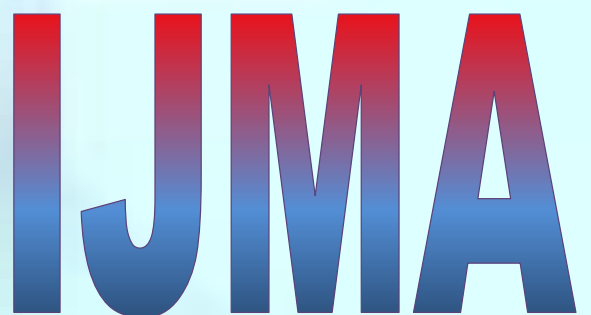

INTERNATIONAL

Journal of MEdical

\section{ARTS}

Volume 3, Issue 1 (Winter 2021)

http://ijma.journals.ekb.eg/

Print ISSN: 2636-4174

Online ISSN: 2682-3780

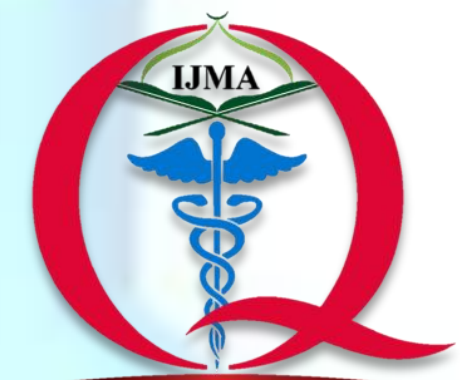

International Jounnal of Medical Arts

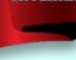




\section{About IJMA}

- International Journal of Medical Arts is the Official Journal of the Damietta Faculty of Medicine, Al-Azhar University, Egypt

- The First Issue was published in July 2019

- It is an International, Open Access, Double-blind, Peerreviewed Journal

- Published four times a year

- Published under the following license: Creative Commons Attribution-ShareAlike 4.0 International Public License (CC BY-SA 4.0). It had updated from the Creative Commons license [CC BY] in volume 2, Issue 4, October 2020

- The Egyptian Knowledge Bank hosts the web site of IJMA

- The Egyptian Knowledge Bank supports IJMA

- IJMA is indexed in the "Directory of Open Access Journals" Indexed on 15 January 2021.

- IJMA follows the regulations of the International Committee of Medical Journal Editors (list date 1/21/20)

- IJMA is a member of The International Society of Managing and Technical Editors

- IJMA is listed in Index Copernicus

- IJMA is listed in Publons, as EKB is an official partner with Clarivate Analytics
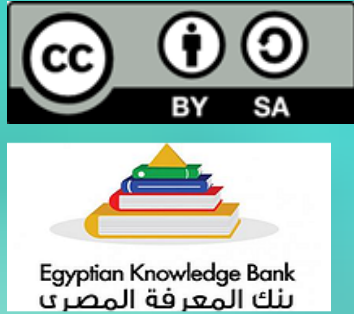

\section{DOAJ}

CMNE

ISMTE

INDEX COPERNICUS

publons 
International Journal of Medical Arts 2021; 3 [1]: 1083-1087.

Available online at Journal Website
https://ijma.journals.ekb.eg/
Main subject [Anesthesia and Intensive Care] ${ }^{*}$

Original article

\title{
Intrathecal Fentanyl versus Dexmedetomedine as Adjuvants to Bupivacaine for Cesarean Section
}

\author{
Tawfik Noor El-Din[1], Mostafa Hussein[2], Ahmed Abd El-galil[1], Ahmed Emad Abdellatif[3] \\ Department of Anesthesia and Intensive Care, Faculty of Medicine, Al-Azhar University, Egypt[1] . \\ Department of Anesthesia and Intensive Care, Faculty of Medicine, Al-Azhar University [Assiut Branch], Egypt[2] \\ Department of Anesthesia and Intensive Care, Damietta Faculty of Medicine, Al-Azhar University, Egypt ${ }^{[3]}$ \\ Corresponding author: Ahmed Emad Abdellatif \\ Email: omda433@gmail.com \\ Received at: Febraury 02, 2020; Revised at: November 23, 2020; Accepted at: November 23, 2020 \\ DOI: 10.21608/ijma.2020.23709.1097
}

\section{ABSTRACT}

Background: Intrathecal a2 agonists prolong the duration of action of local anesthetics and reduce the required dose. Dexmedetomidine is an a2 receptor agonist, and its a2/a1 selectivity is eight times higher than that of clonidine

Aim of the work: This study aimed to determine the effect of adding dexmedetomidine or fentanyl to intrathecal bupivacaine on the onset time, duration, and intensity of motor and sensory blocks as the primary outcome and postoperative analgesia, sedation, and incidence of side effects as a secondary outcome in cesarean section.

Patients and methods: The study was carried out on 40 adults full-term pregnant female submitted for elective cesarean section were randomly classified into two equal groups, twenty patients each: Group [D]: Patients received intrathecally bupivacaine and dexmedetomidine. Group [F]: Patients received intrathecally bupivacaine and fentanyl.

Results: Sensory and motor block onset times were shorter in Group D than in Group F. The regression of the sensory block to S1 dermatome and Bromage 0 were longer in Group D than in Group F. The twodermatome regression time was longer in Group $D$ than in Group $F$. There was a statistically significant decrease in group $F$ regarding systolic, diastolic, mean arterial blood pressure, and heart rate than in group $D$. The postoperative analgesic effect time was longer in group $D$ than in group $F$. Neonatal outcome was normal in all groups

Conclusion: We concluded that adding intrathecal dexmedetomidine to bupivacaine for spinal anesthesia synergistically increase block duration and shortens sensory and motor block onset time with better postoperative analgesia without any significant adverse effects.

Keywords: Spinal anesthesia; Bupivacaine; Caesarean section; Dexmedetomidine; Fentanyl

This is an open-access article registered under the Creative Commons, ShareAlike 4.0 International license [CC BY-SA 4.0] [https://creativecommons.org/licenses/by-sa/4.0/legalcode.

Please cite this article: Noor El-Din T, Hussein M, Abd El-galil A, Abdellatif AE. Intrathecal Fentanyl versus Dexmedetomedine as Adjuvants to Bupivacaine for Cesarean Section. IJMA 2021; 3[1]: 1083-1087. DOI: 10.21608/ijma.2020.23709.1097

${ }^{*}$ Main subject and any subcategories have been classified according to the research topic. 


\section{INTRODUCTION}

In the last two decades, the incidence of elective cesarean section has risen not only in developed countries but also in developing countries ${ }^{[1]}$. With the increasing trend of cesarean section, the spinal block has become the technique of choice due to its rapid onset of action[2]. Despite the advantage of spinal anesthesia as rapid onset and reliability in producing uniform nerve block[3], its main disadvantage is its short duration of action and lack of long-lasting postoperative analgesia[2]. Adequate postoperative analgesia is mandatory to prevent adverse consequences of surgical insult[i] . To overcome these disadvantages and manage postoperative suffering in combining local anesthetic and opioid intrathecal[1]. Such combinations of local anesthesia and opioids have an important synergistic impact, offering strong analgesia with fewer medications and lower side effects. The use of Fentanyl and dexmedetomidine intrathecal can increase intra-and postoperative analgesic actions without serious side effects.

\section{AIM OF THE WORK}

This study aims to determine the efficacy of these combinations on the nerve block, postoperative analgesic effect, and sedation effect.

\section{PATIENTS AND METHODS}

The study was carried out after the local ethics committee in Al-Azhar university of Assiut approval and written informed consent on forty adult full-term pregnant female aged from 21 to 35 years old admitted to Al-Azhar University Hospitals in Assiut and Damietta scheduled for elective cesarean deliveries. According to the ASA [American Society of Anesthesiologist], patients were selected according to the physical status class I to II. The study was carried out from April 2019 to September 2019.

Exclusion criteria were more than ASA status II, $\mathrm{BMI}>30$, patients have an allergy to the study medication, emergency Cesarean Section, twins or more, any fetal anomalies of congenital origin, and an absolute contraindication for spinal anesthesia.

Patients were randomized by computer-generated and closed sealed envelope method into two equal groups, twenty patients each: Group [D]: Patients received intrathecally $2.5 \mathrm{~mL}$ total volume of $10 \mathrm{mg}$
[2 $\mathrm{mL}$ ] $0.5 \%$ hyperbaric bupivacaine [Sunnypivacaine] and $10 \mu \mathrm{g}$ dexmedetomidine in $0.5 \mathrm{~mL}$ [prepared by diluting $1 \mathrm{~mL}$ dexmedetomidine in 5 $\mathrm{mL}$ of normal saline]. Group [F]: Patients received intrathecally $2.5 \mathrm{~mL}$ total volume of $10 \mathrm{mg}[2 \mathrm{~mL}]$ $0.5 \%$ hyperbaric bupivacaine [sunnypivacaine] and $20 \mu \mathrm{g}$ fentanyl in $0.5 \mathrm{~mL}$ [prepared by diluting $2 \mathrm{~mL}$ fentanyl in $2.5 \mathrm{~mL}$ of normal saline].

Every subject had been submitted to a careful pre-anesthesia assessment, including History taking regarding current medical illness, drug therapy, and previous experience with general or regional anesthesia if any. Thorough clinical examination and laboratory investigations: Complete blood picture, Serum creatinine, Prothrombin time, the international normalized ratio [INR], Fasting blood sugar, serum glutamic oxaloacetic transaminase [SGOT], serum glutamicpyruvic transaminase [SGPT].

In the operating theatre, an intravenous cannula [18 G] was inserted, and cases received IV prehydration with $15 \mathrm{~m} / \mathrm{kg}$ Ringer's lactate solution within 20 minutes. All patients received preanesthesia ranitidine $50 \mathrm{mg}$, metoclopramide $10 \mathrm{mg}$ intravenously. Standard monitoring was instituted, including Non-invasive blood pressure, Lead II electrocardiography, and peripheral pulse oximetry $\mathrm{SpO}_{2}$ using [A NIHON KOHDEN monitor]. The drug solutions were prepared by an anesthetist who wasn't involved in the study or care of the patient. Both patient and anesthetist performing the block were blinded to the study drug.

Baseline Pulse rate, blood pressure, and respiratory rate recorded before spinal anesthesia. Under all aseptic precaution, a lumbar puncture was carried out with 25-gauge Quincke's needle [KLAS MEDICAL $\circledast]$ in the L4 L5 or L3 L4 space in sitting position intrathecal injection was given over approximately $10-15$ seconds. After noting the time of injection, the patient was placed in a supine position with $15^{\circ}$ lateral tilt [right hip uppermost] and low flow oxygen [4L/minute] administered via an oxygen mask.

The sensory block [onset time and duration] was assessed by the ice cube method every minute until the level was stabilized for two consecutive tests. Every 10 minutes for an hour and every 30 minutes tell recovery. Time is calculated as the time 
between intrathecal injection and the highest level of sensory block and sensory regression to the $L_{1}$ dermatome recorded. On reaching T7-sensory block level, surgery was permitted. Motor block degree was assessed by Bromage scale[4]: $0=$ No paralysis, 1= lack of ability to elevate the extended leg, 2= lack of ability to flex the knee, $3=$ lack of ability to flex the ankle [full motor blockade]. The regression time to reach sensory or motor block was documented. All calculated times were based on allocating spinal injection time as zero time. When sensory regression reached $\mathrm{S} 1$ dermatome and Bromage 0 [group D 6 \& 1/2 h., group F 4 \& 1/2 h.], patients were discharged to the internal ward. Sedation was recorded by the Ramsay sedation score[5] intraoperative every $5 \mathrm{~min}$. The scale, from 1 to 6 , describes a patient as follows:

Postoperatively, the pain score was recorded by using a visual analog pain scale ${ }^{[6]}$ between 0 and 10 [0 = no pain, $10=$ most severe pain], initially every 1 hour for 2 hours, then every 2 hours for the following 6 hours then every 4 hours till the end of the first postoperative day. All included subjects received [30 mg] intravenous ketorolac at the end of surgery and every 8 hours for 24 hours for postoperative analgesia. Pethidine $20 \mathrm{mg}$ was given intravenously as rescue analgesia when the visual analog scale [VAS] is $\geq 4$ after 2 hours of the administered intravenous ketorolac dose.

Data entry and statistical analyses were performed using SPSS [statistical package of social sciences] version 21 [SPSS Inc., Chicago, IL, USA]. An independent sample t-test [student t-test] was used for regularly distributed results. Statistical significance was considered when the probability $[P]$ value was less than or equal to 0.05 , normally distributed data was defined as mean \pm stander deviation and abnormally median and percentile data.

\section{RESULTS}

Our statistical analysis reveals that the mean age of study groups was $26 \pm 3.03$ for the dexmedetomidine group and $26.5 \pm 4.4$ for the fentanyl group, without a statistically significant difference between both groups [ $p=0.649]$. The sensory block onset was significantly in the dexmedetomidine group than fentanyl group, the mean regression time to the sensory block of two segments was longer in the dexmedetomidine group than the fentanyl group, and the meantime to full sensory recovery was significantly longer in the dexmedetomidine group than fentanyl group [Table 1]. The mean time to achieve Bromage scale 3 motor block was faster in the dexmedetomedine group than the fentanyl group. The time to full motor recovery was longer in the dexmedetomedine group than in the fentanyl group [Table 1]. There was no significant difference between both groups at Ramsey sedation score [Table 3]. The visual analog score was significantly lower in the dexmedetomidine group than the fentanyl group at 2 and 4 hours [Table 4]. The total dose of pethidine [mg] received by patients in the dexmedetomidine group was significantly less than the fentanyl group [Table 5].

Table [1]: Comparison between the studied groups regarding the onset of sensory block, two-segment regression of sensory block, full sensory recovery, onset to reach Bromage 3, and regression to Bromage 0

\begin{tabular}{|l|l|l|l|l|}
\hline \multicolumn{2}{|l|}{} & Group D & Group F & P-value \\
\hline The onset of sensory block [min.] & Mean \pm SD & $1.8 \pm 0.3$ & $2.4 \pm 0.4$ & $\mathbf{0 . 0 0 0 1}$ \\
\hline Bromage [3] to Bromage [0] time [min.] & Mean \pm SD & $336.5 \pm 25.4$ & $242.6 \pm 10.5$ & $\mathbf{0 . 0 0 1}$ \\
\hline
\end{tabular}

Table [2]: Comparison between the two studied groups as regard Ramsey sedation score intraoperatively

\begin{tabular}{|c|c|c|c|c|c|c|c|c|c|c|c|c|c|}
\hline \multicolumn{2}{|c|}{ Time [min.] } & 5 & 10 & 15 & 20 & 25 & 30 & 35 & 40 & 45 & 50 & 55 & 60 \\
\hline \multirow[t]{3}{*}{ Group D } & Min & 2 & 2 & 2 & 2 & 2 & 2 & 2 & 2 & 2 & 2 & 2 & 2 \\
\hline & Max & 2 & 2 & 3 & 3 & 3 & 3 & 3 & 3 & 3 & 3 & 2 & 2 \\
\hline & Median & 2 & 2 & 2 & 2 & 2 & 2 & 2 & 2 & 2 & 2 & 2 & 2 \\
\hline \multirow[t]{4}{*}{ Group F } & Min & 2 & 2 & 2 & 2 & 2 & 2 & 2 & 2 & 2 & 2 & 2 & 2 \\
\hline & Max & 2 & 2 & 3 & 3 & 3 & 3 & 3 & 3 & 3 & 3 & 2 & 2 \\
\hline & Median & 2 & 2 & 2 & 2 & 2 & 2 & 2 & 2 & 2 & 2 & 2 & 2 \\
\hline & $P$ & 1 & 1 & 1 & 0.64 & 1 & 1 & 1 & 1 & 1 & 0.65 & 1 & 1 \\
\hline
\end{tabular}


Table [3]: Comparison between the two studied groups regarding visual analog scale

\begin{tabular}{|l|l|l|l|l|l|l|l|l|c|c|}
\hline \multicolumn{2}{|c|}{ Time [hr.] postoperative } & $\mathbf{1}$ & $\mathbf{2}$ & $\mathbf{4}$ & $\mathbf{6}$ & $\mathbf{8}$ & $\mathbf{1 2}$ & $\mathbf{1 6}$ & $\mathbf{2 0}$ & $\mathbf{2 4}$ \\
\hline \multirow{3}{*}{ Group D } & Min & 0 & 0 & 0 & 0 & 0 & 0 & 1 & 0 & 0 \\
\cline { 2 - 11 } & Max & 0 & 2 & 4 & 4 & 4 & 4 & 4 & 4 & 5 \\
\cline { 2 - 11 } & Median & & 1 & 2 & 2 & 2 & 2 & 2 & 2 & 3 \\
\hline \multirow{5}{*}{ Group F } & Min & 0 & 0 & 0 & 0 & 0 & 0 & 0 & 0 & 0 \\
\cline { 2 - 11 } & Max & 0 & 3 & 5 & 5 & 5 & 4 & 5 & 5 & 5 \\
\cline { 2 - 11 } & Median & & 1 & 3 & 3 & 3 & 2 & 3 & 3 & 3 \\
\hline
\end{tabular}

Table [4]: Total analgesic dose of pethidine in the two studied groups in [mg]

\begin{tabular}{|c|c|c|c|c|}
\hline & & Group D & Group F & P-value \\
\hline \multicolumn{2}{|l|}{ Median } & 20 & 40 & \multirow{6}{*}{0.0001} \\
\hline \multicolumn{2}{|l|}{ Min } & 0 & 20 & \\
\hline \multicolumn{2}{|l|}{ Max } & 40 & 80 & \\
\hline \multirow{3}{*}{ Percentiles } & 25 & 20 & 40 & \\
\hline & 50 & 20 & 40 & \\
\hline & 75 & 40 & 60 & \\
\hline
\end{tabular}

\section{DISCUSSION}

Spinal anesthesia is the most widely applied anesthetic technique for elective cesarean delivery. Postoperative pain is a major challenge. This is due to the shorter time of analgesic action of anesthetic drugs that warranted the use of additional analgesia a short time after operative intervention. In addition, this warrants the use of different adjuvants to increase the duration of postoperative analgesia [e.g., midazolam and clonidine] $]^{[7]}$.

Results of the current study indicated that intrathecal injection of $10 \mathrm{mg}$ of heavy bupivacaine,

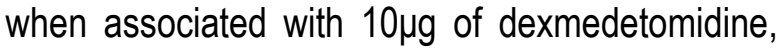
greatly affect the characteristics of spinal anesthesia [e.g., rapid onset of sensory and motor block action] when compared to $20 \mu \mathrm{g}$ fentanyl with heavy bupivacaine, in the spinal block for elective cesarean sections. In addition, dexmedetomidine extended the duration of the block with significant analgesic actions [evidenced by lower VAS scores, reduced need for antalgics, and increased time between surgery and first request of analgesics].

These results were consistent with Gupta et al. [7] Intrathecal [5 $\mu \mathrm{g}$ ] dexmedetomidine was compared with fentanyl [25 $\mu \mathrm{g}$ ] as adjuvants to $12.5 \mathrm{mg}$ hyperbaric bupivacaine in patients organized for sugary of lower abdomen and concluded that intrathecal dexmedetomidine was associated with sustained motor and sensory blockade and reduced demand for rescue analgesics in 24 hours relative to fentanyl. In addition, Bajwa et al.[8] compared dexmedetomidine to fentanyl in epidural analgesia in orthopedics and reported comparable results. The sensory and motor blockade started earlier and lasts longer with a reduction of the postoperative need for analgesia. Dexmedetomidine also had a higher safety profile. Also, Al-Ghanem et al.[9] investigated the effect of the addition of dexmedetomidine to bupivacaine for the spinal block for gynecological surgeries and reported that plain bupivacaine [10 mg] with $5 \mu \mathrm{g}$ dexmedetomidine was associated with significantly long motor and sensory blockade when compared $25 \mu \mathrm{g}$ fentanyl.

The present results were in agreement with that obtained by El-din et al.[10] who studied the effects

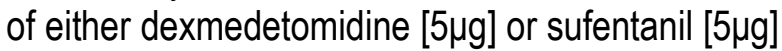
on strong bupivacaine [0.5 percent $10 \mathrm{mg}$ ] for postoperative analgesia effects in cesarean sections and confirmed that dexmedetomidine was extended to two-segment regression, sensory and motor resolution, and time to first rescue analgesics.

Regarding the mean sedation score by Ramsay sedation score in the current research, the patients showed no significant difference in the two groups all the study time. These results were comparable with Al-Mustafa et al. ${ }^{[11]}$, who used $5 \mu \mathrm{g}$ and $10 \mu \mathrm{g}$ of dexmedetomidine in urological procedures applied to spinal bupivacaine did not affect the level of 
consciousness. All patients in both classes had a Ramsay sedation score of 2 [patient cooperative and oriented] and concluded that an increase in the dose of dexmedetomidine did not raise the degree of sedation. In contrast with the results of the present study Chavda et al.[3] and Motiani et al.[12] concluded that the addition of $25 \mu \mathrm{g}$ fentanyl to hyperbaric bupivacaine causes mild sedation.

In the present study, the pain severity assessed by VAS was significantly lower in Group $D$ than in Group $F$ in most of the tests, with a substantial increase in total rescue analgesic intake of pethidine hydrochloride in Group F relative to Group D. Such findings can be clarified by growing the analgesic activity of 5-007-2 adrenergic receptor agonists in pregnant versus non-pregnant women or by suppressing $\mathrm{N}$-methyl-D-aspartate receptor phosphorylation in spinal cornea neurons. This is in line with the report of Gupta et al. [7].

We concluded that intrathecal dexmedetomidine plus bupivacaine for spinal anesthesia synergistically increases block duration and shortens sensory and motor block onset time with improved postoperative analgesia without any significant adverse effects.

Financial and Non-financial Relationships and Activities of Interest

None

\section{REFERENCES}

1. Yeoh SB, Leong SB, Heng AST. Anesthesia for lower-segment cesarean section: Changing perspectives. Indian J Anaesth. 2010;54 [5]:409414. doi:10.4103/ 0019-5049.71037

2. Vyas N, Sahu DK, Parampill R. Comparative study of intrathecal sufentanil bupivacaine versus intrathecal bupivacaine in patients undergoing elective cesarean section. J Anaesthesiol Clin Pharmacol. 2010; 26 [4]: 488-492.

3. Chavda H, Mehta P, Vyas A. A Comparative Study of Intrathecal Fentanyl and Sufentanil with Bupivacaine Heavy for Postoperative Analgesia. Internet J Anesthesiol. 2012;20 [2]: 2-5. doi:10. 5580/1044

4. Craig D, Carli F. Bromage motor blockade score - a score that has lasted more than a lifetime. Can J
Anesth. 2018; 65 [7]: 837-838. doi:10.1007/ s12630-018-1101-7

5. Rasheed AM, Amirah MF, Abdallah M, Parameaswari, Issa M, Alharthy $\mathbf{A}$. Ramsay sedation scale, and Richmond agitation sedation scale: a cross-sectional study. Dimens Crit Care Nurs. 2019; 38 [2]: 90-95. doi:10.1097/DCC. 0000000000000346

6. Delgado DA, Lambert BS, Boutris N, McCulloch PC, Robbins AB, Moreno MR. Validation of digital visual analog scale pain scoring with a traditional paper-based visual analog scale in adults. JAAOS Glob Res Rev. 2018; 2[3]:e088. doi:10.5435/ jaaosglobal-d-17-00088

7. Gupta R, Verma R, Bogra J, Kohli M, Raman R, Kushwaha JK. A comparative study of intrathecal dexmedetomidine and fentanyl as adjuvants to bupivacaine. J Anaesthesiol Clin Pharmacol. 2011; 27 [3]:339-343. doi:10.4103/0970-9185.83678

8. Bajwa SJS, Singh A, Arora V, Kaur J, Parmar S. Comparative evaluation of dexmedetomidine and fentanyl for epidural analgesia in lower limb orthopedic surgeries. Saudi J Anaesth. 2011; 5 [4]: 365. doi:10.4103/1658-354X.87264

9. Al-Ghanem. Effect of Adding Dexmedetomidine versus Fentanyl to Intrathecal Bupivacaine on Spinal Block Characteristics in Gynecological Procedures: A Double-Blind Controlled Study. Am J Appl Sci. 2009; 6 [5]:882-887. doi:10.3844/ ajassp. 2009.882.887

10. El-Din T, Helmy M, Abd-El-Naby M, Al-Ganady S. Motor and sensory blocking effect of intrathecal fentanyl versus dexmedetomidine as adjuvants to bupiva-caine for cesarean section. Sci J Al-Azhar Med Fac Girls 2018; 2[1]:6. doi:10.4103/sjamf. sjamf_4_18

11. Al-Mustafa MM, Abu-Halaweh SA, Aloweidi AKS, Murshidi MM, Ammari BA, Awwad ZM, et al. Effect of dexmedetomidine added to spinal bupivacaine for urological procedures. Saudi Med J. 2009; 30 [3]:365-370. PMID: 19271064

12. Motiani $P$, Chaudhary $S$, Bahl N, Sethi AK. Intrathecal sufentanil versus fentanyl for lower limb surgeries - a randomized controlled trial. J Anesthesiol Clin Pharmacol. 2011;27[1]:67-73. PMID: 21804710. 


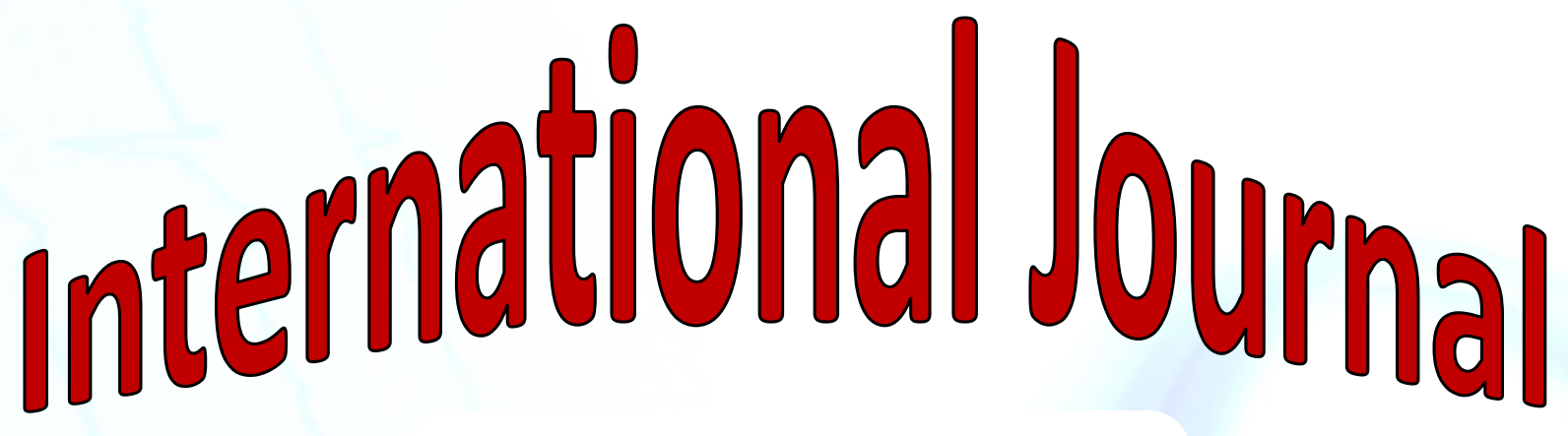

https://ijma.journals.ekb.eg/

Print ISSN: 2636-4174

Online ISSN: 2682-3780

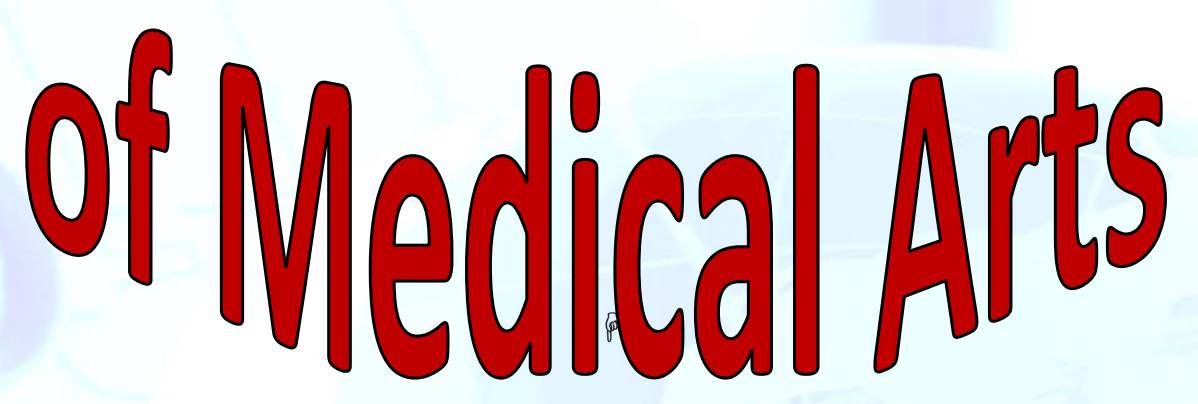

